DOI: $10.15393 /$ j3.art.2015.2629

UDC $517.51,517.58$

K. Nantomah, E. Prempeh

\title{
CERTAIN INEQUALITIES INVOLVING THE $Q$-DEFORMED GAMMA FUNCTION
}

\begin{abstract}
This paper is inspired by the work of J. Sándor in 2006. In the paper, the authors establish some double inequalities involving the ratio $\frac{\Gamma_{q}(x+1)}{\Gamma_{q}\left(x+\frac{1}{2}\right)}$, where $\Gamma_{q}(x)$ is the $q$-deformation of the classical Gamma function denoted by $\Gamma(x)$. The method employed in presenting the results makes use of Jackson's $q$-integral representation of the $q$-deformed Gamma function. In addition, Hölder's inequality for the $q$-integral, as well as some basic analytical techniques involving the $q$-analogue of the psi function are used. As a consequence, $q$-analogues of the classical Wendel's asymptotic relation are obtained. At the end, sharpness of the inequalities established in this paper is investigated.
\end{abstract}

Key words: Gamma function, q-deformed Gamma function, q-integral, inequality

2010 Mathematical Subject Classification: 33B15, 33D05

1. Introduction and Preliminaries. Let $\Gamma(x)$ be the well-known classical Gamma function defined for $x>0$ by

$$
\Gamma(x)=\int_{0}^{\infty} t^{x-1} e^{-t} d t
$$

The psi function $\psi(x)$, otherwise known as the digamma function, is defined as the logarithmic derivative of the Gamma function. That is,

$$
\psi(x)=\frac{d}{d x} \ln (\Gamma(x))=\frac{\Gamma^{\prime}(x)}{\Gamma(x)}, \quad x>0 .
$$

(C) Petrozavodsk State University, 2015 
The Jackson's $q$-integrals from 0 to $a$ and from 0 to $\infty$ are defined as follows [1]:

$$
\begin{gathered}
\int_{0}^{a} f(t) d_{q} t=(1-q) a \sum_{n=0}^{\infty} f\left(a q^{n}\right) q^{n} \\
\int_{0}^{\infty} f(t) d_{q} t=(1-q) \sum_{-\infty}^{\infty} f\left(q^{n}\right) q^{n}
\end{gathered}
$$

provided that the sums converge absolutely.

In a generic interval $[a, b]$, the Jackson's $q$-integral takes the following form:

$$
\int_{a}^{b} f(t) d_{q} t=\int_{0}^{b} f(t) d_{q} t-\int_{0}^{a} f(t) d_{q} t
$$

For more information on this special integral, see [1].

For $a \in C$, the set of complex numbers, we have the following notations: $(a ; q)_{0}=1, \quad(a ; q)_{n}=\prod_{i=0}^{n-1}\left(1-a q^{i}\right), \quad(a ; q)_{\infty}=\prod_{i=0}^{\infty}\left(1-a q^{i}\right)$ and $[n]_{q} !=\frac{(q ; q)_{n}}{(1-q)^{n}}$

The $q$-deformed Gamma function (also known as the $q$-Gamma function or the $q$-analogue of the Gamma function) is defined for $q \in(0,1)$ and $x>0$ by

$$
\begin{aligned}
\Gamma_{q}(x) & =\int_{0}^{\frac{1}{1-q}} t^{x-1} E_{q}^{-q t} d_{q} t=\int_{0}^{[\infty]_{q}} t^{x-1} E_{q}^{-q t} d_{q} t= \\
& =(1-q)^{1-x} \prod_{n=0}^{\infty} \frac{1-q^{n+1}}{1-q^{n+x}},
\end{aligned}
$$

where $E_{q}^{t}=\sum_{n=0}^{\infty} q^{\frac{n(n-1)}{2}} \frac{t^{n}}{[n]_{q} !}=(-(1-q) t ; q)_{\infty}$ is a $q$-analogue of the classical exponential function. See also [2, [3, [4, [5] and the references therein. 
The function $\Gamma_{q}$ exhibits the following properties (see [3]

$$
\begin{aligned}
\Gamma_{q}(x+1) & =[x]_{q} \Gamma_{q}(x), \\
\Gamma_{q}(1) & =1 \\
\Gamma_{q}\left(\frac{1}{2}\right) & =\sqrt{\pi_{q}}
\end{aligned}
$$

where $[x]_{q}=\frac{1-q^{x}}{1-q}$, and $\pi_{q}=q^{\frac{1}{4}}\left(\left[-\frac{1}{2}\right]_{q^{2}} !\right)^{2}$ is the $q$-analogue of $\pi$. Note that $\pi_{q}$ is obtained by setting $n=0$ in the $q$-factorial $[n]_{q}$ !.

Let $\psi_{q}(x)$ be the $q$-analogue of the psi function similarly defined for $x>0$ as follows

$$
\psi_{q}(x)=\frac{\Gamma_{q}^{\prime}(x)}{\Gamma_{q}(x)}=-\ln (1-q)+\ln q \sum_{n=0}^{\infty} \frac{q^{n+x}}{1-q^{n+x}} .
$$

It is well-known in literature that this function is increasing for $x>0$. For instance, see Lemma 2.2 of [6].

In 1987, Lew, Frauenthal and Keyfitz [7] by studying certain problems of traffic flow established the double inequality:

$$
2 \Gamma\left(n+\frac{1}{2}\right) \leq \Gamma\left(\frac{1}{2}\right) \Gamma(n+1) \leq 2^{n} \Gamma\left(n+\frac{1}{2}\right), \quad n \in N .
$$

The inequalities (3) can be rearranged as follows:

$$
\frac{2}{\sqrt{\pi}} \leq \frac{\Gamma(n+1)}{\Gamma\left(n+\frac{1}{2}\right)} \leq \frac{2^{n}}{\sqrt{\pi}}
$$

In 2006, Sándor 8] by using the following inequalities, proved by Wendel 9

$$
\left(\frac{x}{x+s}\right)^{1-s} \leq \frac{\Gamma(x+s)}{x^{s} \Gamma(x)} \leq 1
$$

for $x>0$ and $s \in(0,1)$, extended and refined inequality (3) as follows

$$
\sqrt{x} \leq \frac{\Gamma(x+1)}{\Gamma\left(x+\frac{1}{2}\right)} \leq \sqrt{x+\frac{1}{2}}
$$


for $x>0$.

The objective of this paper is to establish certain inequalities involving the $q$-deformed Gamma function. First, employing similar techniques as in [8, 9, and [10], we prove an $q$-analogue of the double inequality (5). Next, using basic analytical procedures, we prove some related double inequality. At the end, we investigate the sharpness of the inequalities established.

2. Main Results. Let us begin with the following Lemma.

Lemma 1. Assume that $s \in(0,1)$ and $q \in(0,1)$. Then for any $x>0$ the following inequality is valid.

$$
\left(\frac{[x]_{q}}{[x+s]_{q}}\right)^{1-s} \leq \frac{\Gamma_{q}(x+s)}{[x]_{q}^{s} \Gamma_{q}(x)} \leq 1 .
$$

Proof. We employ the Hölder's inequality for the Jackson's $q$-integral:

$$
\int_{0}^{\infty} f(t) g(t) d_{q} t \leq\left[\int_{0}^{\infty}(f(t))^{a} d_{q} t\right]^{\frac{1}{a}}\left[\int_{0}^{\infty}(g(t))^{b} d_{q} t\right]^{\frac{1}{b}},
$$

where $\frac{1}{a}+\frac{1}{b}=1$ and $a>1$.

Let $a=\frac{1}{1-s}, \quad b=\frac{1}{s}, \quad f(t)=t^{(1-s)(x-1)} E_{q}^{-(1-s) q t}$ and $g(t)=t^{s x} E_{q}^{-s q t}$. Then the Hölder's inequality implies

$$
\begin{aligned}
\Gamma_{q}(x+s)= & \int_{0}^{\frac{1}{1-q}} t^{x+s-1} E_{q}^{-q t} d_{q} t \leq \\
\leq & {\left[\int_{0}^{\frac{1}{1-q}}\left(t^{(1-s)(x-1)} E_{q}^{-(1-s) q t}\right)^{\frac{1}{1-s}} d_{q} t\right]^{1-s} \times } \\
& \times\left[\int_{0}^{\frac{1}{1-q}}\left(t^{s x} E_{q}^{-s q t}\right)^{\frac{1}{s}} d_{q} t\right]^{s}= \\
= & {\left[\int_{0}^{\frac{1}{1-q}} t^{x-1} E_{q}^{-q t} d_{q} t\right]^{1-s}\left[\int_{0}^{\frac{1}{1-q}} t^{x} E_{q}^{-q t} d_{q} t\right]^{s}=} \\
= & {\left[\Gamma_{q}(x)\right]^{1-s}\left[\Gamma_{q}(x+1)\right]^{s} . }
\end{aligned}
$$

Thus,

$$
\Gamma_{q}(x+s) \leq\left[\Gamma_{q}(x)\right]^{1-s}\left[\Gamma_{q}(x+1)\right]^{s}
$$


Substituting (2) into (7) we obtain

$$
\Gamma_{q}(x+s) \leq\left[\Gamma_{q}(x)\right]^{1-s}[x]_{q}^{s}\left[\Gamma_{q}(x)\right]^{s},
$$

which implies

$$
\Gamma_{q}(x+s) \leq[x]_{q}^{s} \Gamma_{q}(x) .
$$

Substitution $s$ by $1-s$ in (8) gives

$$
\Gamma_{q}(x+1-s) \leq[x]_{q}^{1-s} \Gamma_{q}(x) .
$$

Substitution $x$ by $x+s$ results to

$$
\Gamma_{q}(x+1) \leq[x+s]_{q}^{1-s} \Gamma_{q}(x+s) .
$$

Now, combining inequalities (8) and (10), we obtain

$$
\frac{\Gamma_{q}(x+1)}{[x+s]_{q}^{1-s}} \leq \Gamma_{q}(x+s) \leq[x]_{q}^{s} \Gamma_{q}(x),
$$

which can be written as

$$
\frac{[x]_{q}}{[x+s]_{q}^{1-s}} \Gamma_{q}(x) \leq \Gamma_{q}(x+s) \leq[x]_{q}^{s} \Gamma_{q}(x) .
$$

Finally, (11) can be rearranged as:

$$
\left(\frac{[x]_{q}}{[x+s]_{q}}\right)^{1-s} \leq \frac{\Gamma_{q}(x+s)}{[x]_{q}^{s} \Gamma_{q}(x)} \leq 1,
$$

which concludes the proof of Lemma 1 .

Theorem 1. Assume that $q \in(0,1)$. Then the inequality

$$
\sqrt{[x]_{q}} \leq \frac{\Gamma_{q}(x+1)}{\Gamma_{q}\left(x+\frac{1}{2}\right)} \leq \sqrt{\left[x+\frac{1}{2}\right]_{q}}
$$

is valid for any $x>0$.

Proof. By setting $s=\frac{1}{2}$ in the $q$-analogue of the Wendel's inequalities (6), we get

$$
\frac{1}{\sqrt{[x]_{q}}} \leq \frac{\Gamma_{q}(x)}{\Gamma_{q}\left(x+\frac{1}{2}\right)} \leq \frac{\sqrt{\left[x+\frac{1}{2}\right]_{q}}}{[x]_{q}} .
$$


Using (2), we can arrange 13 as follows:

$$
\sqrt{[x]_{q}} \leq \frac{\Gamma_{q}(x+1)}{\Gamma_{q}\left(x+\frac{1}{2}\right)} \leq \sqrt{\left[x+\frac{1}{2}\right]_{q}}
$$

Remark 1. Inequalities (6) imply

$$
\lim _{x \rightarrow \infty} \frac{\Gamma_{q}(x+s)}{[x]_{q}^{s} \Gamma_{q}(x)}=1 .
$$

Remark 2. Since $[x]_{q}^{\beta-\alpha} \frac{\Gamma_{q}(x+\alpha)}{\Gamma_{q}(x+\beta)}=\frac{\Gamma_{q}(x+\alpha)}{[x]_{q}^{\alpha} \Gamma_{q}(x)} \cdot \frac{[x]_{q}^{\beta} \Gamma_{q}(x)}{\Gamma_{q}(x+\beta)}$, then using 14) we obtain

$$
\lim _{x \rightarrow \infty}[x]_{q}^{\beta-\alpha} \frac{\Gamma_{q}(x+\alpha)}{\Gamma_{q}(x+\beta)}=1, \quad \alpha, \beta \in(0,1) .
$$

Remark 3. The equalities (14), (15) are the q-analogues of the classical Wendel's asymptotic relation [9]:

$$
\lim _{x \rightarrow \infty} \frac{\Gamma(x+s)}{x^{s} \Gamma(x)}=1
$$

Theorem 2. Assume that $q \in(0,1)$ is fixed. Then the inequalities

$$
\frac{1}{\sqrt{\pi_{q}}}<\frac{\Gamma_{q}(x+1)}{\Gamma_{q}\left(x+\frac{1}{2}\right)}<(1+\sqrt{q}) \cdot \frac{1}{\sqrt{\pi_{q}}}
$$

are valid for $x \in(0,1)$.

Proof. Define a function $U(q, x)$ for $q \in(0,1)$ and $x \geq 0$ by

$$
U(q, x)=\frac{\Gamma_{q}(x+1)}{\Gamma_{q}\left(x+\frac{1}{2}\right)} .
$$

Notice that $\Gamma_{q}(1)=\Gamma_{q}(2)=1, \quad \Gamma_{q}\left(\frac{1}{2}+1\right)=\left[\frac{1}{2}\right]_{q} \Gamma_{q}\left(\frac{1}{2}\right)=\left[\frac{1}{2}\right]_{q} \sqrt{\pi_{q}}$, $\left[\frac{1}{2}\right]_{q}=\frac{1-\sqrt{q}}{1-q}, \quad U(q, 0)=\frac{1}{\sqrt{\pi_{q}}}$ and $U(q, 1)=(1+\sqrt{q}) \cdot \frac{1}{\sqrt{\pi_{q}}}$.

Now let $f(q, x)=\ln U(q, x)$. Then

$$
f(q, x)=\ln \frac{\Gamma_{q}(x+1)}{\Gamma_{q}\left(x+\frac{1}{2}\right)}=\ln \Gamma_{q}(x+1)-\ln \Gamma_{q}\left(x+\frac{1}{2}\right) .
$$


For a fixed $q \in(0,1)$, we obtain

$$
f^{\prime}(q, x)=\psi_{q}(x+1)-\psi_{q}\left(x+\frac{1}{2}\right)>0,
$$

since $\psi_{q}(x)$ is increasing for $x>0$. Hence, $U(q, x)=e^{f(q, x)}$ is increasing on $x>0$, and for $x \in(0,1)$ we have $U(q, 0)<U(q, x)<U(q, 1)$ establishing (17).

Remark 4. Define $F$ by $F(q, x)=[x]_{q}^{-\frac{1}{2}} \frac{\Gamma_{q}(x+1)}{\Gamma_{q}\left(x+\frac{1}{2}\right)}$ for $q \in(0,1)$ and $x>0$. Let $g(x)=\ln F(q, x)=\ln \Gamma_{q}(x+1)-\ln \Gamma_{q}\left(x+\frac{1}{2}\right)-\frac{1}{2} \ln [x]_{q}$. Then, $g^{\prime}(x)=\psi_{q}(x+1)-\psi_{q}\left(x+\frac{1}{2}\right)+\frac{1}{2} \frac{q^{x} \ln q}{1-q^{x}}$. The following Stieltjes integral representations are valid

$$
\psi_{q}(x)=-\ln (1-q)-\int_{0}^{\infty} \frac{e^{-x t}}{1-e^{-t}} d \mu_{q}(t), \quad \int_{0}^{\infty} e^{-x t} d \mu_{q}(t)=-\frac{q^{x} \ln q}{1-q^{x}}
$$

where $\mu_{q}(t)=-\ln q \sum_{k=1}^{\infty} \delta(t+k \ln q)$ and $\delta$ represents the Dirac delta function. See [11] and the references therein. Then

$$
\begin{aligned}
g^{\prime}(x) & =-\int_{0}^{\infty} \frac{e^{-(x+1) t}}{1-e^{-t}} d \mu_{q}(t)+\int_{0}^{\infty} \frac{e^{-\left(x+\frac{1}{2}\right) t}}{1-e^{-t}} d \mu_{q}(t)-\frac{1}{2} \int_{0}^{\infty} e^{-x t} d \mu_{q}(t) \\
& =\int_{0}^{\infty} \frac{e^{-x t}}{1-e^{-t}} \phi(t) d \mu_{q}(t)
\end{aligned}
$$

where $\phi(t)=e^{-\frac{1}{2} t}-\frac{1}{2} e^{-t}-\frac{1}{2}<0$. By the Hausdorff-Bernstein-Widder theorem (see [12] and the references therein), we obtain $g^{\prime}(x)<0$, so $g(x)$ is strictly deacreasing. Consequently, $F(q, x)$ is strictly decreasing. Hence, $F(q, x) \geq \lim _{x \rightarrow \infty} F(q, x)=1$ yielding the lower bound of (12).

Remark 5. Define $G$ by $G(q, x)=\left[x+\frac{1}{2}\right]_{q}^{-\frac{1}{2}} \frac{\Gamma_{q}(x+1)}{\Gamma_{q}\left(x+\frac{1}{2}\right)}$ for $q \in(0,1)$ and $x>0$. Let $w(x)=\ln G(q, x)=\ln \Gamma_{q}(x+1)-\ln \Gamma_{q}\left(x+\frac{1}{2}\right)-\frac{1}{2} \ln \left[x+\frac{1}{2}\right]_{q}$. Then, $w^{\prime}(x)=\psi_{q}(x+1)-\psi_{q}\left(x+\frac{1}{2}\right)+\frac{1}{2} \frac{q^{x+\frac{1}{2}} \ln q}{1-q^{x+\frac{1}{2}}}$. By setting $a=\frac{1}{2}, b=1$, $c=\frac{1}{2}$ and $k=1$ in Theorem 7.2 of [13], we obtain, $\psi_{q}(x+1)-\psi_{q}\left(x+\frac{1}{2}\right) \geq-\frac{1}{2} \frac{q^{x+\frac{1}{2}} \ln q}{1-q^{x+\frac{1}{2}}}$.

Consequently, $w^{\prime}(x) \geq 0$, so $w(x)$ is increasing. As a result, $G(q, x)$ is also increasing. Hence, $G(q, x) \leq \lim _{x \rightarrow \infty} G(q, x)=1$ yielding the upper bound of (12). 
Remark 6. Let $H(q, x)=\frac{\sqrt{\pi_{q}} \Gamma_{q}(x+1)}{\Gamma_{q}\left(x+\frac{1}{2}\right)}$. Then, $H(q, x)$ is increasing, and for $x \in(0,1)$ we have,

$1=\lim _{x \rightarrow 0^{+}} H(q, x) \leq H(q, x)$ and $H(q, x) \leq \lim _{x \rightarrow 1^{-}} H(q, x)=1+\sqrt{q}$ respectively yielding the lower and upper bounds of (17).

Based on the above remarks, the estimates in (12) and (17) are sharp.

Acknowledgment. The authors are very grateful to the anonymous referees for their useful comments and suggestions, which helped in improving the quality of this paper.

\section{References}

[1] Jackson F. H. On a q-definite integrals. Quarterly Journal of Pure and Applied Mathematics, 1910, vol. 41, pp. 193-203.

[2] Askey R. The q-Gamma and q-Beta Functions. Applicable Anal., 1978, vol. 8, no. 2, pp. 125-141.

[3] Chung W. S., Kim T. and Mansour T. The q-deformed Gamma function and q-deformed Polygamma function. Bull. Korean Math. Soc., 2014, vol. 51, no. 4, pp. 1155-1161.

[4] Ege I., Yýldýrým E. Some generalized equalities for the q-gamma function. Filomat, 2012, vol. 12, no. 6, pp. 1221-1226. DOI: 10.2298/FIL1206227E.

[5] Elmonster H., Brahim K., Fitouhi A. Relationship between characterizations of the q-Gamma function. Journal of Inequalities and Special Functions, 2012, vol. 3, no. 4, pp. 50-58.

[6] Shabani A. S. Generalization of some inequalities for the q-gamma function. Annales Mathematicae et Informaticae, 2008, vol. 35, pp. 129-134.

[7] Lew J., Frauenthal J., Keyfitz N. On the average distances in a circular disc. Mathematical Modeling: Classroom Notes in Applied Mathematics, Philadelphia, SIAM, 1987.

[8] Sándor J. On certain inequalities for the Gamma function. RGMIA Res. Rep. Coll., 2006, vol. 9, no. 1, Art. 11.

[9] Wendel J. G. Note on the gamma function. Amer. Math. Monthly, 1948, vol. 55, pp. 563-564.

[10] Qi F., Luo Q. M. Bounds for the ratio of two Gamma functions - from Wendel's and related inequalities to logarithmically completely monotonic functions. Banach Journal of Mathematical Analysis, 2012, vol. 6, no. 2, pp. $123-158$. 
[11] Ismail M. E. H. and Muldoon M. E. Inequalities and monotonicity properties for Gamma and q-Gamma functions. Available online at: http://arxiv.org/pdf/1301.1749v1.pdf.

[12] Mortici C. A sharp inequality involving the psi function. Acta Universitatis Apulensis, 2010, vol. 22, pp. 41-45.

[13] Qi F. Bounds for the ratio of two Gamma functions. Journal of Inequalities and Applications, vol. 2010, Art ID 493058.

Received November 5, 2014.

In revised form, June 15, 2015.

University for Development Studies

P. O. Box 24, Navrongo, UE/R, Ghana

E-mail: mykwarasoft@yahoo.com, knantomah@uds.edu.gh

Kwame Nkrumah University of Science and Technology

Kumasi, Ghana

E-mail: eprempeh.cos@knust.edu.gh 